\title{
Assessment of the relationship between stenosis severity and distribution of coronary artery stenoses on multislice computed tomographic angiography and myocardial ischemia detected by single photon emission computed tomography
}

\author{
Balaji K. Tamarappoo, PhD, MD, ${ }^{\mathrm{a}, \mathrm{b}}$ Ariel Gutstein, MD, ${ }^{\mathrm{c}}$ Victor Y. Cheng, MD, ${ }^{\mathrm{a}, \mathrm{b}, \mathrm{d}}$ \\ Ryo Nakazato, MD, , ${ }^{\mathrm{a}, \mathrm{b}}$ Heidi Gransar, MS, ${ }^{\mathrm{a}, \mathrm{b}}$ Damini Dey, PhD, ${ }^{\mathrm{a}, \mathrm{b}, \mathrm{d}}$ \\ Louise E. J. Thomson, MBChB, FRACP, ${ }^{\mathrm{a}, \mathrm{b}, \mathrm{d}}$ Sean W. Hayes, MD, ${ }^{\mathrm{a}, \mathrm{b}, \mathrm{d}}$ \\ John D. Friedman, MD, ${ }^{\mathrm{a}, \mathrm{b}, \mathrm{d}}$ Guido Germano, PhD, ${ }^{\mathrm{a}, \mathrm{b}, \mathrm{d}}$ Piotr J. Slomka, PhD, ${ }^{\mathrm{a}, \mathrm{b}, \mathrm{d}}$ \\ and Daniel S. Berman, MD ${ }^{a, b, d}$
}

Background. The relationship between luminal stenosis measured by coronary CT angiography (CCTA) and severity of stress-induced ischemia seen on single photon emission computed tomographic myocardial perfusion imaging (SPECT-MPI) is not clearly defined. We sought to evaluate the relationship between stenosis severity assessed by CCTA and ischemia on SPECT-MPI.

Methods and Results. ECG-gated CCTA (64 slice dual source CT) and SPECT-MPI were performed within 6 months in 292 patients (ages 26-91, 73\% male) with no prior history of coronary artery disease. Maximal coronary luminal narrowing, graded as $\mathbf{0 ,} \geq \mathbf{2 5 \%}, \mathbf{5 0 \%}, \mathbf{7 0 \%}$, or $90 \%$ visual diameter reduction, was consensually assessed by two expert readers. Perfusion defect on SPECT-MPI was assessed by computer-assisted visual interpretation by an expert reader using the standard 17 segment, 5 point-scoring model (stress perfusion defect of $\geq 5 \%$ = abnormal). By SPECT-MPI, abnormal perfusion was seen in $46 / 292$ patients. With increasing stenosis severity, positive predictive value (PPV) increased $(42 \%, 51 \%$, and $74 \%$, $P=.01)$ and negative predictive value was relatively unchanged $(97 \%, 95 \%$, and $91 \%)$ in detecting perfusion abnormalities on SPECT-MPI. In a receiver operator curve analysis, stenosis of $50 \%$ and $70 \%$ were equally effective in differentiating between the presence and absence of ischemia. In a multivariate analysis that included stenosis severity, multivessel disease, plaque composition, and presence of serial stenoses in a coronary artery, the strongest predictors of ischemia were stenosis of $50-89 \%$, odds ratio $(O R) 7.31, P=.001$, stenosis $\geq 90 \%$, OR 34.05, $P=.0001$, and serial stenosis $\geq 50 \%$ OR of $3.55, P=.006$.

Conclusions. The PPV of CCTA for ischemia by SPECT-MPI rises as stenosis severity increases. Luminal stenosis $\geq \mathbf{9 0 \%}$ on CCTA strongly predicts ischemia, while $<50 \%$ stenosis strongly predicts the absence of ischemia. Serial stenosis of $\geq \mathbf{5 0 \%}$ in a vessel may offer incremental value in addition to stenosis severity in predicting ischemia. ( $J$ Nucl Cardiol 2010;17:791-802.)

From the Department of Imaging (Division of Nuclear Medicine) ${ }^{a}$ Department of Medicine (Division of Cardiology), and CSMC Burns \& Allen Research Institute, ${ }^{\mathrm{b}}$ Cedars-Sinai Medical Center, Los Angeles, CA; Department of Cardiology, ${ }^{\mathrm{c}}$ Rabin Medical Center, Petach-Tikva, Israel; and Department of Medicine, ${ }^{\mathrm{d}}$ University of California at Los Angeles, School of Medicine, Los Angeles, CA.

Balaji K. Tamarappoo and Ariel Gutstein contributed equally to this work.

The study was supported in part by grants from the Lincy Foundation and Diane and Gilford Glazer.
Received for publication Dec 29, 2009; final revision accepted Mar 28, 2010.

Reprint requests: Daniel S. Berman, MD, Department of Imaging (Division of Nuclear Medicine), Cedars-Sinai Medical Center, 8700 Beverly Boulevard, Room 1258, Los Angeles, California 90048; bermand@cshs.org.

$1071-3581 / \$ 34.00$

Copyright $@ 2010$ The Author(s). This article is published with open access at Springerlink.com

doi:10.1007/s12350-010-9230-6 
Key Words: Myocardial perfusion imaging $\cdot$ SPECT $\cdot$ computed tomography (CT) • ischemia $\cdot$ myocardial $\cdot$ SPECT $\cdot$ coronary artery disease

\section{INTRODUCTION}

Coronary CT angiography (CCTA) provides excellent anatomic assessment of the extent and severity of coronary artery disease (CAD), with high diagnostic accuracy for detection of significant stenosis in patients who present with symptoms suggestive of ischemia. ${ }^{1-4}$ With its high negative predictive value, CCTA has become useful for exclusion of CAD in patients with chest pain syndromes. However, the clinical management of a patient with an abnormal CCTA is not well defined. Single photon emission computed tomography (SPECT) myocardial perfusion imaging (MPI) is an accepted standard for detection and quantification of significant myocardial ischemia and has been shown to be a strong prognostic indicator of the risk for adverse cardiac events. ${ }^{5,6}$ The increasing use of CCTA for diagnosis of $\mathrm{CAD}$ has led to several studies comparing stenosis severity on CCTA to ischemia on SPECT-MPI ${ }^{7-11}$ and PET. ${ }^{12}$ However, these reports have been limited by modest-sized, heterogeneous populations that often included patients known to have CAD. Furthermore, previous studies have not addressed the relationship between CCTA-based assessment of plaque characteristics and ischemia by SPECT-MPI. In this study, we compared CCTA-based assessment of stenosis severity to SPECTMPI in a large cohort of consecutive patients with no previous history of CAD. Our objectives were to determine whether there is a degree of luminal stenosis on CCTA above which myocardial ischemia on SPECT-MPI is highly likely, to determine the degree of stenosis severity below which abnormal SPECT-MPI is so unlikely that further diagnostic testing would not be required, and to explore whether the distribution of significant stenoses in the vessel can improve prediction of myocardial ischemia.

\section{METHODS}

\section{Patients and Study Protocol}

We studied 294 consecutive patients without known CAD_-defined by history of myocardial infarction, coronary revascularization, or presence of angiographically documented coronary stenoses of $\geq 50 \%$ luminal diameter by invasive coronary angiography-participating in the Cedars-Sinai Cardiac Imaging Database Registry and undergoing both CCTA and SPECT-MPI for suspected ischemic heart disease at Cedars-Sinai Medical Center within 6 months. These patients did not experience change in symptoms or coronary revascularization between the examinations. Two patients with excessive motion artifact on CCTA were excluded. Pre-test likelihood of CAD before the first test performed was determined according to the Diamond and Forrester method using
$<15 \%,>85 \%$, and $15-85 \%$ for low, high, and intermediate pre-test likelihood, respectively. ${ }^{13,14}$ The study protocol was approved by the Cedars-Sinai Institutional Review Board, and all patients included in the study provided informed consent for the use of their clinical and imaging data.

\section{CCTA Image Acquisition}

CCTA was performed on a SOMATOM Definition dualsource computed tomography scanner (Siemens Medical Systems, Forchheim, Germany) which employs a flying focus along the $z$-axis with two different focal spots, acquiring 64 overlapping .6-mm image slices with a temporal resolution of $83 \mathrm{~ms} .{ }^{15}$ The CCTA protocol has been previously described in detail. ${ }^{16}$ Unless contraindicated, a sublingual spray of $.4 \mathrm{mg}$ nitroglycerin (Sciele Pharma, Alpharetta, Georgia) was administered and $80 \mathrm{~mL}$ of intravenous contrast (Omnipaque or Visipaque, GE Healthcare, Princeton, New Jersey) was injected into the antecubital vein at $5 \mathrm{~mL} \cdot \mathrm{s}^{-1}$, followed by $80 \mathrm{~mL}$ of saline at $5 \mathrm{~mL} \cdot \mathrm{s}^{-1}$. ECG-gated scanning from $1 \mathrm{~cm}$ below the tracheal bifurcation to the level of the diaphragm was performed in a single breath-hold. Scanning parameters included heart ratedependent pitch (.2 to .45), $330 \mathrm{~ms}$ gantry rotation time, 100 or $120 \mathrm{kVp}$ tube voltage, and $600 \mathrm{mAs}$ tube current. Dose modulation protocols were implemented according to our previously described methods. ${ }^{17}$ The effective radiation dose in the patients, using the various acquisition protocols, was $11.3 \pm 6.4 \mathrm{mSv}$.

\section{CCTA Image Reconstruction}

Retrospectively gated reconstruction of raw CCTA data was performed at $40 \%$ (when available), $65 \%, 70 \%, 75 \%$, and $80 \%$ of the R-R interval using the following parameters: $.6-\mathrm{mm}$ slice thickness $\left(.75 \mathrm{~mm}\right.$ if $\left.\mathrm{BMI}>35 \mathrm{~kg} \cdot \mathrm{m}^{-2}\right), .3-\mathrm{mm}$ slice increment, 250 -mm field-of view, $512 \times 512$ matrix, and B26f "medium smooth" kernel or a B46f "sharp" kernel in patients with dense coronary calcium (calcium score $>100$ ).

\section{CCTA Image Interpretation}

Reconstructed data were transferred to a Siemens workstation (Leonardo; Siemens Medical solutions) or a Hewlett-Packard workstation (Palo Alto, California) running the Vitrea 2 clinical software (Vital Images, Minnetonka, Minnesota). CCTA examinations were read for this research by two experienced CCTA readers (B. T. and R. N.) blinded to patient clinical status, SPECTMPI, and clinically reported CCTA findings. Observers visually assessed each coronary segment using standard axial images, oblique multiplanar reformations, and oblique maximum intensity projections. ${ }^{18}$ Twenty-five coronary segments including the left main (LM) were analyzed and for the purpose of per-vessel analysis, the maximal stenosis from each of the three major coronary arteries were used. ${ }^{19}$ With respect to branching vessels, the diagonal and ramus intermediate branch were combined with the 
left anterior descending artery (LAD), the obtuse marginal (OM) branch combined with the left circumflex (LCX) and the posterolateral and posterior descending artery combined with the right coronary artery (RCA) or the LCX depending on whether they originated from the RCA or LCX. Maximal diameter stenosis severity was visually graded by consensus between the two observers on a scale of 0 to $6(0=0 \%, 1=1 \%$ to $24 \%, 2=25 \%$ to $49 \%, 3=50 \%$ to $69 \%, 4=70 \%$ to $89 \%, 5=90 \%$ to $99 \%$, and $6=100 \%) .{ }^{20}$ We had demonstrated previously that visual stenosis assessment by this grading system and quantitative CTbased stenosis assessment were equivalent when compared to stenosis quantification by invasive angiography. ${ }^{20}$ Plaque was further characterized as calcified or non-calcified plaque as described previously. ${ }^{21}$ When plaque contained both calcified and non-calcified components with neither of them constituting $>75 \%$ of the plaque volume it was described as a partially calcified or mixed plaque. Data were analyzed after excluding all patients with densely calcified plaque for per-patient analysis and after excluding all vessels with dense calcification for per-vessel analysis. A secondary analysis was performed by including all patients and vessels with dense calcification. For this analysis the artery with dense calcification obscuring the coronary lumen was considered to have a maximal luminal stenosis $\geq 90 \%$. For assignment of plaque distribution within individual vessels, the ramus intermediate branch was considered an extension of the proximal LAD, the diagonal branch an extension of the mid-LAD, the OM branches an extension of the proximal circumflex and the PDA and posterolateral branches an extension of the distal RCA or distal LCX depending on coronary dominance. For quantification of coronary artery calcium, all CT images were reviewed by an expert reader, using semi-automatic commercially available software (ScImage, Los Altos, CA). Total Agatston CCS was calculated as the sum of calcified plaque scores of all coronary arteries. $^{22}$

\section{Exercise and Adenosine Stress SPECT-MPI Protocols}

Patients were instructed to discontinue beta-blockers and calcium antagonists 48 hours and nitrates 24 hours before testing. Rest perfusion images were acquired 10 minutes after infusion of 7-9 mCi of ${ }^{99 \mathrm{~m}} \mathrm{Tc}$-sestamibi or $3-4.5 \mathrm{mCi}$ of ${ }^{201} \mathrm{Tl}$ (based on body weight). Stress testing was performed with a symptom-limited Bruce treadmill exercise protocol or vasodilator challenge, as described previously. ${ }^{23}$ At near-maximal exercise, ${ }^{99 \mathrm{~m}} \mathrm{Tc}$-sestamibi (32-40 $\mathrm{mCi}$ based on patient weight) was injected intravenously, after which treadmill exercise was continued at maximal workload for 1 minute and at one stage lower for two additional minutes whenever possible. ${ }^{99 \mathrm{~m}} \mathrm{Tc}$ sestamibi MPI acquisition was started 15-30 minutes after radiopharmaceutical injection. For vasodilator stress, adenosine was infused at $140 \mu \mathrm{g} \cdot \mathrm{kg}^{-1} \cdot \min ^{-1}$ for 5 minutes and in ambulatory patients, a low-level treadmill exercise was performed during adenosine infusion. ${ }^{24}$ At the end of the second minute, ${ }^{99 \mathrm{~m}} \mathrm{Tc}$-sestamibi $(32-40 \mathrm{mCi})$ was injected and myocardial imaging was started approximately 60 minutes later. ${ }^{6}$ Twelve lead electrocardiography (ECG) was monitored continuously during stress testing. Horizontal or downsloping
ST-segment depression $\geq 1 \mathrm{~mm}$ or upsloping $\geq 1.5 \mathrm{~mm}$ were considered positive for ischemia.

\section{SPECT-MPI Acquisition and Reconstruction Protocols}

SPECT images were acquired with a 2-detector gamma camera (Philips Adac Forte or Vertex, Philips Medical Systems, Cleveland, Ohio or E-Cam, Siemens Medical Solutions). Highresolution collimators were used, and acquisition consisted of 64 projections over a $180^{\circ}$ orbit, with 64 projections at $25 \mathrm{~s} /$ projection for supine ${ }^{99 \mathrm{~m}} \mathrm{Tc}$ acquisition followed immediately by 15 s/projection for prone ${ }^{99 \mathrm{~m}} \mathrm{Tc}$ acquisition. ${ }^{25}$ Rest $^{201} \mathrm{Tl}$ acquisition was performed at $35 \mathrm{~s} /$ projection in supine position only. At each of the 64 projection angles, the image data were recorded into 16 equal ECG-gated time bins. No attenuation or scatter correction was applied. After iterative reconstruction (12 iterations) with Butterworth prefiltering (cutoff, .66 cycle/pixel for supine ${ }^{99 \mathrm{~m}} \mathrm{Tc}$, .55 cycle/pixel for prone ${ }^{99 \mathrm{~m}} \mathrm{Tc}$; order 5), short-axis images were automatically generated. ${ }^{26}$

\section{Automated Quantification of SPECT MPI}

All SPECT MPI studies were reanalyzed quantitatively for purposes of this research study, using an objective, automatic computer analysis. ${ }^{27}$ Automatically generated myocardial contours were evaluated by a core laboratory technologist without knowledge of any clinical data, and when necessary, contours were adjusted to correspond to the myocardium ( $8 \%$ of cases). A qualitative assessment of image quality using a visual 5-point scale $(1=$ uninterpretable, $2=$ poor, $3=$ fair, $4=$ good, and $5=$ excellent) was performed. The quantitative perfusion variable employed was total perfusion deficit (TPD), which is a validated, computer-derived analog of the percent myocardium abnormal by visual analysis, representing both extent and severity of perfusion defect. ${ }^{28}$ TPD was calculated as the percentage of the total surface area of the left ventricle below the predefined uniform average deviation threshold using QPS software. ${ }^{27}$ TPD was measured at stress and rest, and patients with a stress TPD of $\geq 5 \%$ were considered abnormal and were considered to represent myocardial ischemia in these patients with no known CAD. ${ }^{28,29} \mathrm{~A}$ computer-derived maximum severity score was also quantified using QPS software and was expressed on a scale of 0-4 $(0=$ normal and $4=$ absence of tracer uptake $)$ for each patient and for each coronary territory. ${ }^{27}$ Defects in the anterior and septal wall were allocated to the left anterior descending coronary artery (LAD); in the lateral wall, the left circumflex coronary artery (LCX); in the inferior wall, right coronary artery (RCA). When the assignment of a perfusion defect to a vascular territory was not clear (watershed regions), we used automated fusion software described previously and assigned the vessel according to the large vessel supplying the region in question. ${ }^{30}$

\section{Statistical Analysis}

Continuous variables were expressed with median and range. Categorical variables were expressed as percentages. The severity of luminal stenosis, presence perfusion defects by 
SPECT-MPI, and frequency of perfusion defects were considered categorical variables. The Wilcoxon sum test, and a Kruskall Wallis test were used to compare continuous variables and Pearson Chi-square tests were used to compare ordinal variables. Sensitivity, specificity, and other diagnostic test measures were used to compare the different cut points of degree of stenosis to predict ischemia. Receiver operating characteristic (ROC) curves were constructed to assess the discriminatory value of various CCTA stenosis cutoffs in detecting the presence and absence of inducible ischemia by SPECT-MPI. The measured areas under the ROC curves were compared as described by Hanley and McNeil. ${ }^{31}$ CCTA-based imaging features of coronary artery stenosis including luminal stenosis severity, plaque composition, number of coronary arteries with stenosis $\geq 50 \%$, and the presence of serial stenoses $\geq 50 \%$ were included in a stepwise fashion in a multivariable logistic regression analysis and adjusted for baseline clinical risk factors to determine the strongest significant predictors of ischemia. A $P$-value of $<.05$ was considered significant. Data were analyzed using STATA software version 9 (www.stata.com).

\section{RESULTS}

\section{Patient Characteristics}

Of the 292 patients, 192 were men $(66 \%$, see Table 1). The median age was 62 years (range, 26-91), and the median pretest likelihood of CAD (before the first test) was $58 \%$ (range, 2-96\%). Seventy percent $(n=203)$ of patients had chest discomfort or shortness of breath before the first test that persisted at the time of the second test. The remaining 30\% $(n=89)$ had either equivocal or abnormal results on the first test $(n=43)$, or discordant results (e.g., abnormal stress ECG, normal SPECT-MPI) $(n=27)$. In seven patients, the results of the first test resulted in a higher post-test likelihood prompting a second test and in 12 patients the reason for the second test was not clear. The duration between studies ranged from 0 to 178 days with a median of 4 days. Overall, in $75 \%$ of patients SPECT-MPI and CCTA were performed within 2 weeks.

\section{SPECT-MPI Findings}

Exercise stress testing was used in $201(68 \%)$ of patients, of whom $97 \%$ achieved $>85 \%$ of maximal predicated heart rate. Adenosine stress was employed in the remaining $91(32 \%)$ patients. Stress perfusion abnormalities were seen in 46 patients $(15.4 \%)$. In patients with abnormal perfusion, stress TPD ranged from $5 \%$ to $48 \%$ with a median defect of $14 \%$ and ischemic TPD (Stress TPD-Rest TPD) ranged from $4 \%$ to $16 \%$ with a median defect of $6 \%$. The proportion of patients with stress perfusion abnormalities was similar between pharmacologic and exercise stress. Perfusion

Table 1. Characteristics of patients included in the analysis

\begin{tabular}{|c|c|c|}
\hline & Normal SPECT-MPI & Abnormal SPECT-MPI \\
\hline Number of patients & 246 & 46 \\
\hline $\operatorname{Age}^{*}(\mathrm{y})$ & $26-86(61)$ & $31-91(72)$ \\
\hline Body Mass Index* $\left(\mathrm{kg} \cdot \mathrm{m}^{-2}\right)$ & $15.1-43.2(26.6)$ & $17.6-48.7(27.3)$ \\
\hline Men & $161(65 \%)$ & $31(67 \%)$ \\
\hline Women & $85(35 \%)$ & $15(33 \%)$ \\
\hline Diabetes & $22(9 \%)$ & $6(12 \%)$ \\
\hline Hypertension & $96(39 \%)$ & $21(46 \%)$ \\
\hline Hyperlipidemia & $121(49 \%)$ & $27(58 \%)$ \\
\hline History of Smoking & $39(16 \%)$ & $6(12 \%)$ \\
\hline Claudication & $3(1 \%)$ & $1(2 \%)$ \\
\hline Pre-test likelihood* & $.02-.98(.59)$ & $.05-.98(.58)$ \\
\hline Low likelihood & $96(39 \%)$ & $14(31 \%)$ \\
\hline Intermediate likelihood & $91(37 \%)$ & $17(36 \%)$ \\
\hline High likelihood & $59(24 \%)$ & $15(33 \%)$ \\
\hline \multicolumn{3}{|l|}{ Reasons for testing: } \\
\hline Angina & 47 (19\%) & $12(26 \%)$ \\
\hline Atypical chest pain & $81(33 \%)$ & $12(27 \%)$ \\
\hline Dyspnea & $79(32 \%)$ & $17(36 \%)$ \\
\hline Other symptoms & $39(16 \%)$ & $5(11 \%)$ \\
\hline Duration between CCTA and SPECT* (days) & $0-178(4)$ & $0-172(8)$ \\
\hline
\end{tabular}

*Values expressed as range (median). 
defects were localized to the LAD in 29 patients, LCX in 13 patients and RCA in 17 patients. There were 12 patients with stress-induced perfusion defects in more than 1 vascular territory.

\section{CCTA Findings}

CCTA study quality was excellent or good in 283 studies $(97 \%)$ and fair in 6 studies. Calcium scores ranged from 0 to 4305 with a median score of 35 . Nine patients had dense calcification in one or more coronary arteries were excluded from the primary analysis. A secondary analysis was performed by considering these patients to have a maximal stenosis of $\geq 90 \%$. Ninetyfour patients $(32 \%)$ had no detectable coronary artery plaque, $104(35 \%)$ had plaque causing a maximal luminal stenosis of $>0 \%$ and $<50 \%$ of the vessel diameter, 58 (20\%) had a maximal stenosis of $\geq 50 \%$ and $<90 \%$, and $27(9 \%)$ had a maximal stenosis of $\geq 90 \%$. In 27 patients, two or more coronary arteries exhibited luminal stenosis of $\geq 70 \%$. Maximal stenosis was found in a proximal segment in $54 \%$ of patients, in a mid segment in $16 \%$, in a distal segment in $6 \%$, and in multiple locations in the same vessel (serial stenoses) in $24 \%$. In patients with any detectable plaque, calcified plaque caused the maximal stenosis in $42 \%$, partially calcified plaque in $41 \%$, and non-calcified plaque in $17 \%$.

On a per-vessel basis, 473 arteries were found to have no stenosis, 271 had a maximal stenosis of $>0 \%$ and $<50 \%$, 80 had a maximal stenosis of $\geq 50 \%$ and $<90 \%$, and 38 had a maximal stenosis of $\geq 90 \%$. There were 13 coronary arteries with dense calcium in which luminal stenosis could not be reliably assessed by both expert readers.

\section{Comparison of Stenosis Severity by CCTA and Ischemia on SPECT-MPI}

With increasing grades of stenosis severity from $0 \%$ to $100 \%$, there was an increase in the proportion of individuals with abnormal myocardial perfusion by SPECT-MPI, $P=.001$ (Figure 1). Only a small proportion of patients with $<50 \%$ stenosis on CCTA had stress perfusion defects, including three of 94 patients (3\%) without coronary artery plaque (stress TPD 5-6\%) (Figure 1) and three of 59 patients (5\%) with atherosclerotic plaque and a maximal luminal narrowing of $1-24 \%$ (stress TPD 5-8\%). A progressive increase in the frequency of ischemia was observed in the $>50 \%$ stenosis categories. Among patients with a luminal stenosis severity of $50-70 \%, 17 \%$ were found to have perfusion abnormalities by SPECT-MP increasing to $42 \%$ and $74 \%$ when maximal stenosis was $70-89 \%$ and $90-100 \%$, respectively (Figure 1).

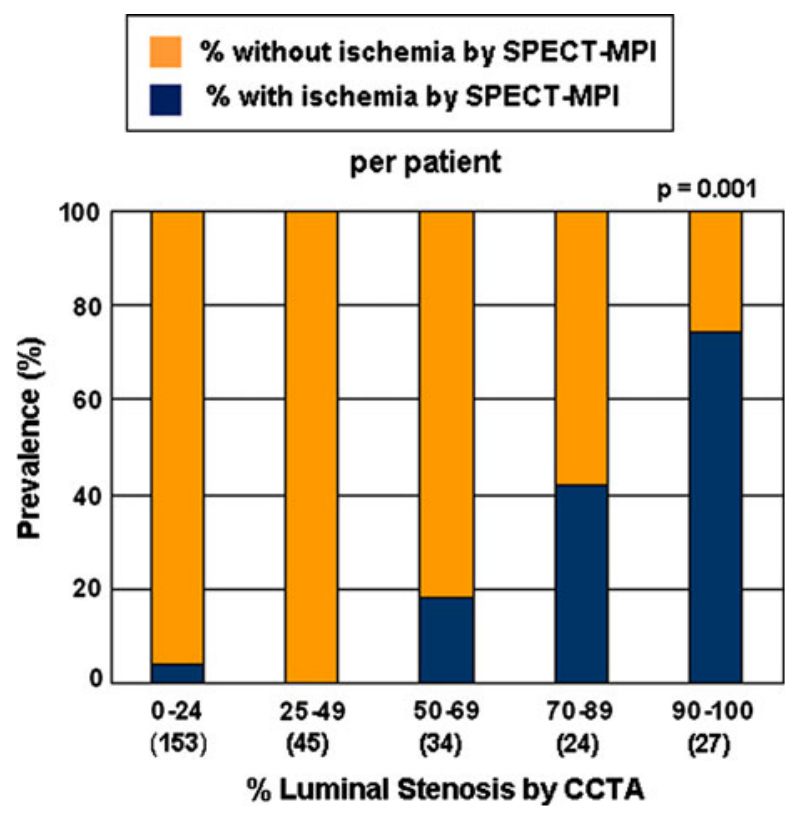

Figure 1. Prevalence of ischemia on SPECT-MPI in patient groups defined by maximal stenosis detected during CCTA. Bars represent percent of individuals in each stenosis category with ischemia, defined by TPD $\geq 5 \%$, on SPECT-MPI. The numbers in parentheses represent number of individuals in each stenosis category. In a per-patient analysis, prevalence of ischemia increased in proportion to stenosis severity with $P=.001$ across all categories of stenosis severity.

Among all patients with luminal stenosis of $0-24 \%$, 25-49\%, and 50-69\%, mean TPD was low, .05 \pm .31 , $.12 \pm .48$, and $.72 \pm 1.14$, respectively, $P=\mathrm{ns}$ (Figure 2). There was a significant increase in the mean stress TPD to $3.2 \pm 3.5$ and $12.9 \pm 12.84$ when stenosis severity increased to $50-89 \%$ and $90-100 \%$ (Figure 2). Similarly, when the mean severity of stress perfusion defects alone was considered, there was no significant difference in severity scores in patients with maximal luminal narrowing of $0-24 \%, 25-49 \%$, and $50-69 \%(.19 \pm .31$, $.001 \pm .0$, and $.29 \pm .48$, respectively, $P=$ ns (Figure 3 ). When maximal luminal stenosis increased to the $70-89 \%$ and $90-100 \%$ categories, there was a proportional increase in the severity of stress perfusion abnormality to $.72 \pm .14$ and $2.58 \pm 1.34$, respectively $(P<.001$ and $P<.0001$, respectively) (Figure 3 ). While the stenosis category $50-69 \%$ was usually not associated with ischemia, $17 \%$ of these patients manifested stress-induced ischemia on SPECT-MPI as noted above.

The positive predictive value (PPV) of CCTA for abnormal stress perfusion increased in proportion to the severity of luminal stenosis, $42 \%, 51 \%$, and $74 \%$ for $<50 \%$ stenosis, $50-89 \%$ stenosis, and $\geq 90 \%$ stenosis, respectively, $P=.01$ (Table 2 ) with only a minor change in NPV $(97 \%, 95 \%$, and 91\%). Similar results 


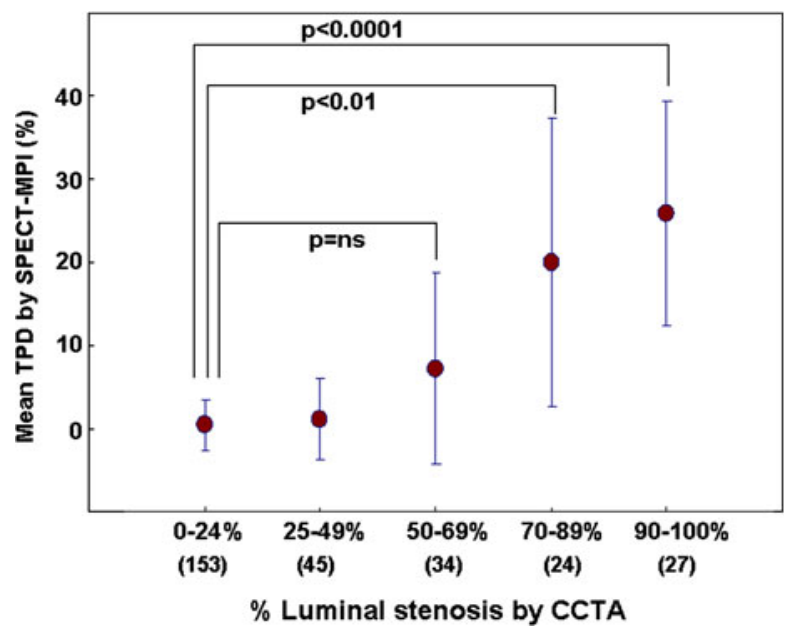

Figure 2. Comparison of maximal stenosis detected by CCTA and TPD quantified by SPECT-MPI and expressed as percent perfusion defect on a continuous scale. Mean and standard deviation of the TPD for patients in each stenosis category are shown. On a per-patient basis, TPD increases with increase in maximal stenosis severity with $P<.0001$ across all categories of stenosis severity and $P<.001$ when patients with stenosis severity of all categories except $90-100 \%$ are compared.

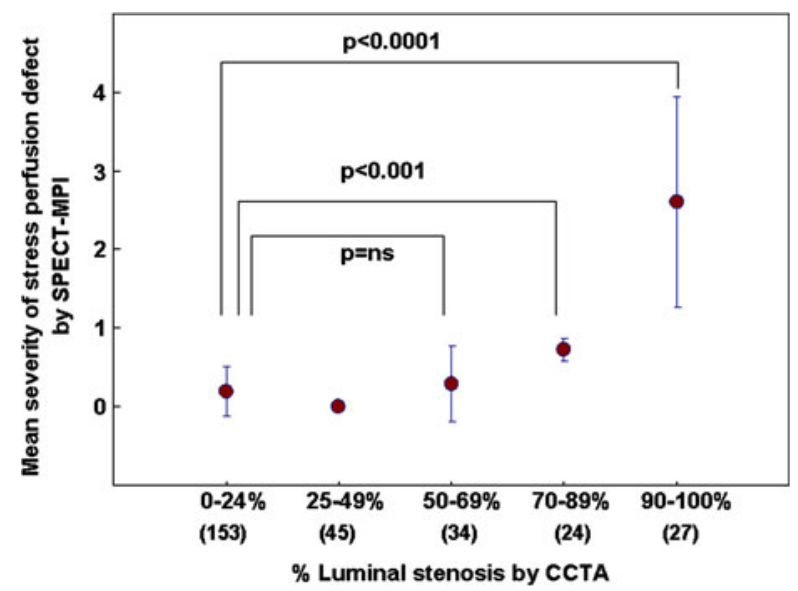

Figure 3. Comparison of maximal stenosis detected by CCTA and the severity of ischemia quantified by SPECT-MPI and expressed on a scale of 0-4. Mean and standard deviation of the severity of ischemia for patients in each stenosis category are shown. On a per-patient basis, the severity of ischemia increases with increase in maximal stenosis severity with $P<.0001$ across all categories of stenosis severity and $P<.001$ when patients with stenosis severity of all categories except $90-100 \%$ are compared.

were obtained in a per-vessel analysis; increasing degrees of luminal stenosis resulted in a similar increase in PPV, $38 \%, 51 \%$, and $68 \%$ for $<50 \%$ stenosis, $50-89 \%$ stenosis, and $\geq 90 \%$ stenosis, respectively, $P=.001$. Whereas, NPV remained unchanged $(98 \%, 98 \%$, and
97\%) (Table 2). When patients with vessels containing dense calcification were included in a secondary analysis, there was still a progressive increase in the PPV of CCTA for stress perfusion defect with increasing severity of luminal stenosis on both a per-patient and per-vessel basis and the NPV remained relatively unchanged (Table 2).

On a per-patient basis, there was a strong association between the coronary artery with the maximal luminal stenosis and the region of myocardium affected by ischemia: 40 (87\%) patients exhibited stress-induced ischemia in territories perfused by arteries with luminal stenosis of maximal severity that ranged from $50 \%$ to $100 \%$. An example of a patient with a $70-89 \%$ stenosis in the proximal RCA with a stress perfusion defect in the corresponding vascular territory is shown in Figure 4. There were 12 patients with stress-induced perfusion defects in more than one vascular territory. In three of these patients, the ischemic territory was in a watershed region that could have been ascribed to the LCX or the RCA. In these patients the assignment of the perfusion territory was based on fusion imaging and coronary dominance.

In six patients, luminal stenosis was absent or minimal (0-24\%) in the vessels that corresponded to the ischemic territories. The quality of SPECT-MPI images in five of these patients was suboptimal from motion or breast attenuation artifact and they were unable to undergo prone imaging. A sixth patient was found to have severe left ventricular hypertrophy by CCTA without significant coronary artery stenosis.

\section{ROC Analysis of Stenosis Severity Predictive of Ischemia by SPECT-MPI}

On a per-patient basis, the areas under the curve (AUC) were significantly higher for both the $50 \%$ stenosis and $70 \%$ stenosis cut-offs, compared to the $90 \%$ stenosis cut-off $(.825$ and .816 , respectively, vs .725 , $P=.01$, Figure 5). There was no significant difference between the AUC seen with $50 \%$ and $70 \%$ stenosis cut-offs.

\section{Univariable Analysis of CCTA-Based Determinants of Ischemia}

Univariable analysis was used to identify variables that were associated with ischemia among patients with coronary artery stenosis. In addition to traditional risk factors such as increasing age, male gender, hypertension, hyperlipidemia, and diabetes, the severity of luminal stenosis, presence of multiple vessels containing $\geq 50 \%$ stenoses and serial $\geq 50 \%$ stenoses in one coronary artery were strongly associated with ischemia. 
Table 2. Accuracy of CCTA stenosis severity for detection of ischemia by SPECT-MPI

\begin{tabular}{|c|c|c|c|c|}
\hline & Sensitivity & Specificity & PPV & NPV \\
\hline \multicolumn{5}{|l|}{ Patient based analysis } \\
\hline Maximal stenosis $\geq 50 \%$ & $86(70-94)$ & $80(74-84)$ & $42(32-54)$ & $97(93-99)$ \\
\hline Maximal stenosis $\geq 70 \%$ & $65(46-80)$ & $91(87-94)$ & $51(36-66)$ & $95(91-97)$ \\
\hline Maximal stenosis $\geq 90 \%$ & $48(32-63)$ & $97(94-99)$ & $74(53-88)$ & $91(87-94)$ \\
\hline $\mathrm{Chi}^{2}=9.37, P=.01$ & & & & \\
\hline \multicolumn{5}{|c|}{ Patient based analysis including individuals with vessels containing dense calcium } \\
\hline Maximal stenosis $\geq 50 \%$ & 87(73-94) & $78(72-82)$ & $43(33-53)$ & $97(93-99)$ \\
\hline Maximal stenosis $\geq 70 \%$ & $74(58-85)$ & $89(85-92)$ & $57(43-69)$ & $95(91-97)$ \\
\hline Maximal stenosis $\geq 90 \%$ & $52(37-66)$ & $95(91-97)$ & $67(49-81)$ & $91(87-94)$ \\
\hline \multicolumn{5}{|l|}{$\mathrm{Chi}^{2}=6.97, P=.03$} \\
\hline \multicolumn{5}{|l|}{ Vessel based analysis } \\
\hline Maximal stenosis $\geq 50 \%$ & $83(70-92)$ & $91(89-93)$ & $38(29-48)$ & $98(97-99)$ \\
\hline Maximal stenosis $\geq 70 \%$ & $68(54-80)$ & $96(94-97)$ & $51(39-63)$ & $98(97-99)$ \\
\hline Maximal stenosis $\geq 90 \%$ & $48(35-62)$ & $99(97-99)$ & $68(51-81)$ & $97(95-98)$ \\
\hline $\mathrm{Chi}^{2}=18, P=.001$ & & & & \\
\hline \multicolumn{5}{|c|}{ Vessel based analysis including vessels with dense calcium } \\
\hline Maximal stenosis $\geq 50 \%$ & $85(73-92)$ & $90(88-92)$ & $38(30-47)$ & $99(98-99)$ \\
\hline Maximal stenosis $\geq 70 \%$ & $71(58-82)$ & $95(93-96)$ & $49(38-60)$ & $98(97-99)$ \\
\hline $\begin{array}{l}\text { Maximal stenosis } \geq 90 \% \\
\mathrm{Chi}^{2}=9.37, P=.01\end{array}$ & $53(39-65)$ & $98(96-98)$ & $60(46-73)$ & $97(95-98)$ \\
\hline
\end{tabular}

$95 \%$ confidence intervals are shown in parentheses.
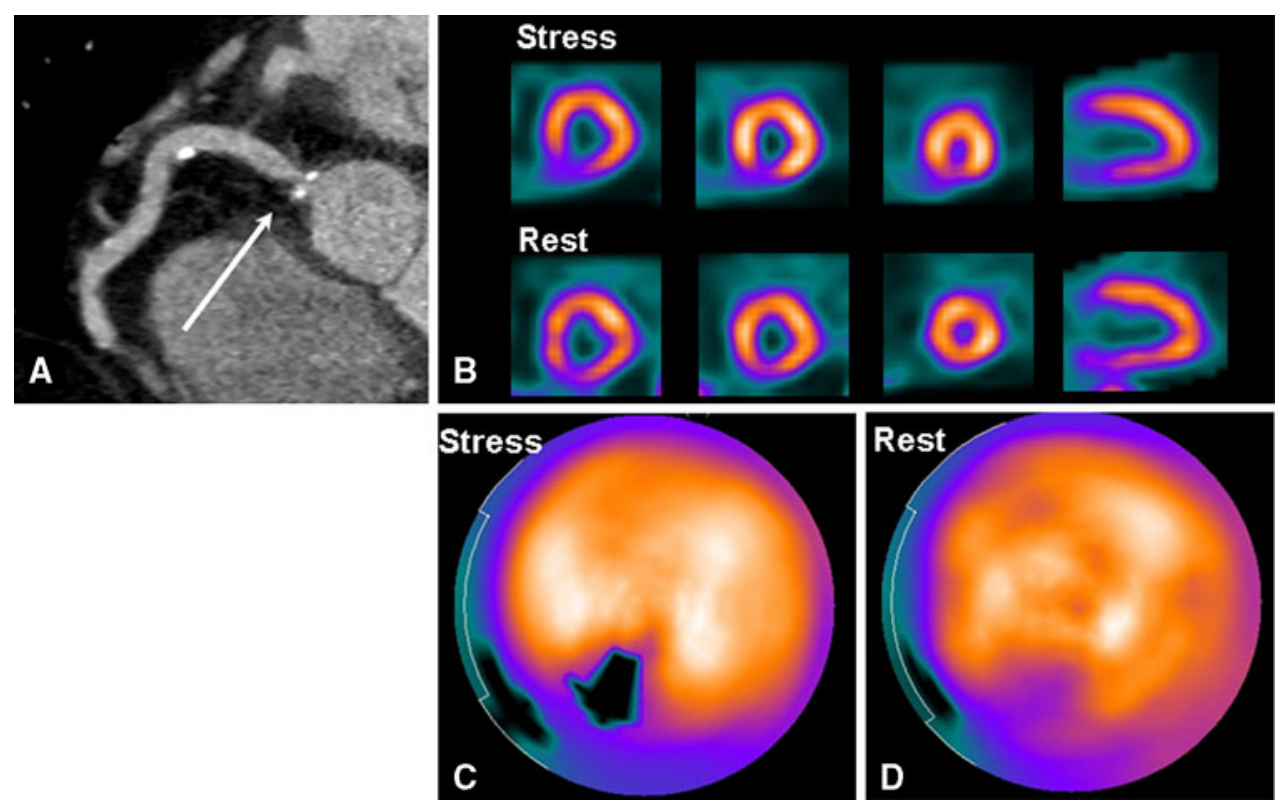

Figure 4. An example of stress-induced ischemia in a 58-year-old male with a $70-89 \%$ stenosis in the proximal RCA with a non-calcified plaque. (A) A $2.5 \mathrm{~mm}$ thick maximum intensity projection (MIP) of a CCTA showing the RCA. The arrow points to the partially calcified plaque causing $70-89 \%$ stenosis at the ostium of the RCA. (B) Stress and rest SPECT-MPI showing a reversible perfusion defect in this same patient in the mid and distal inferior wall. (C, D) Polar maps of stress and rest SPECT-MPI showing the reversible perfusion defect in the RCA territory. 


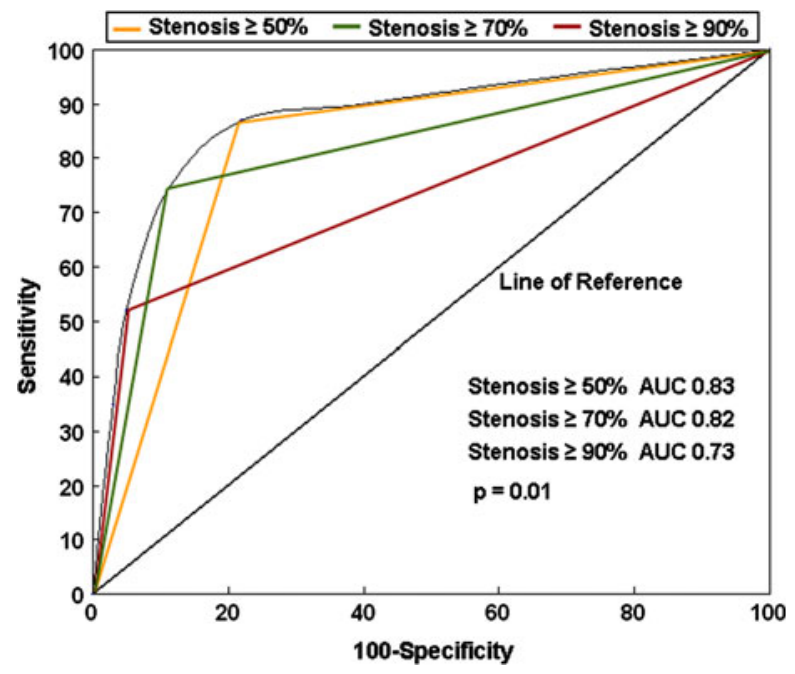

Figure 5. ROC curves representing the diagnostic accuracy of CCTA-based stenosis severity thresholds for identifying individuals with ischemia by SPECT-MPI. The AUC for $50 \%$, $70 \%$, and $90 \%$ stenosis cut-offs were $.83, .82$, and .73 , respectively, with a $\mathrm{Chi}^{2}=8.6$ and $P=.01$ for comparison across all stenosis thresholds.

\section{Association of Plaque Location with Ischemia}

Among patients with stenosis of $\geq 50 \%$, prevalence of ischemia was significantly different between patients, based on whether plaque $\geq 50 \%$ was located in the proximal, mid, distal, or multiple segments of the vessel (Table 3A). The odds of ischemia increased 4.2 fold (95\% confidence interval $(95 \% \mathrm{CI}) 1.7-10.1, P=.002)$ when serial $\geq 50 \%$ stenoses were identified in a single vessel (Table $3 \mathrm{~A}$ ). When patients with $>90 \%$ stenosis were excluded, a strong association between serial stenoses $\geq 50 \%$ and the prevalence of ischemia still remained (Table 3B). The odds of ischemia increased 4.4-fold (95\% CI 1.68-11.7, $P=.004$ ) when serial $\geq 50 \%$ stenoses were identified in a single vessel in patients with a maximal stenosis of $50-90 \%$.

\section{Relationship Between Plaque Characteristics and Ischemia}

When the association of ischemia was compared between patients with single vessel stenosis vs multivessel stenoses, patients with $\geq 50 \%$ stenosis in two or more vessels were more likely to have ischemia when compared to individuals with stenosis in a single vessel (Table 4). When the relationship between plaque composition and ischemia was evaluated, ischemia was more commonly associated with non-calcified or partially calcified plaque when compared to calcified plaque. In a univariable model, on a per patient basis, the odds of
Table 3. Relationship between plaque location and ischemia on SPECT-MPI

\begin{tabular}{|c|c|c|c|}
\hline $\begin{array}{l}\text { Plaque location } \\
\text { in patients with } \\
\text { stenosis } \geq 50 \%\end{array}$ & $\begin{array}{l}\text { Odds } \\
\text { ratio }\end{array}$ & $\boldsymbol{P}$ & $95 \%$ CI \\
\hline \multicolumn{4}{|l|}{ A } \\
\hline Proximal & .40 & .06 & $.21-.93$ \\
\hline Mid & .26 & .1 & $.10-1.32$ \\
\hline Distal & .99 & .6 & $.24-4.70$ \\
\hline Multiple & 4.21 & $.002 *$ & $1.73-10.11$ \\
\hline $\begin{array}{l}\text { Plaque location } \\
\text { in patients with } \\
\text { stenosis } 50-89 \%\end{array}$ & $\begin{array}{l}\text { Odds } \\
\text { ratio }\end{array}$ & $\boldsymbol{P}$ & $95 \% \mathrm{CI}$ \\
\hline \multicolumn{4}{|l|}{$\mathbf{D}$} \\
\hline Proximal & .41 & .06 & $.15-1.11$ \\
\hline Mid & .17 & .08 & $.02-1.42$ \\
\hline Distal & .88 & .63 & $.15-5.17$ \\
\hline Multiple & 4.40 & $.004^{\dagger}$ & $1.68-11.17$ \\
\hline
\end{tabular}

*Univariate analysis describing increased odds of ischemia with serial stenoses $\geq 50 \%$ in the same vessel in patients with maximal stenosis $\geq 50 \%$. $P<.05$ is statistically significant.

Univariate analysis describing increased odds of ischemia with serial stenoses $\geq 50 \%$ in the same vessel in patients with maximal stenosis $\geq 50 \%$ and $<90 \%$. $P<.05$ is statistically significant.

ischemia was greater when the maximal stenosis was caused by non-calcified plaque (OR of $4.89,95 \%$ CI $1.05-22.73, P=.04)$; however, partially calcified and calcified plaque were not strongly associated with ischemia (Table 4).

\section{Multivariable Model of CCTA-Based Predictors of Ischemia}

For identification of CCTA predictors of ischemia, a multivariable model was designed to include degrees of stenosis severity $(<50 \%, 50-89 \%$, and $\geq 90 \%)$, plaque composition (calcified, non-calcified, and partially calcified plaque), stenosis $\geq 50 \%$ in multiple vessels and location of stenosis $\geq 50 \%$ (proximal, mid, distal, or serial stenoses). When this model was applied to all 292 patients, the most significant predictor of ischemia was stenosis severity with an OR of 7.31, 95\% CI of 2.35$22.70, P=.001$, for $50-89 \%$ stenosis, and OR of 34.05 , $95 \%$ CI of $10.60-109.34, P=.0001$, for stenosis $\geq 90 \%$. Serial stenosis of $\geq 50 \%$ remained significant as a predictor of ischemia with an OR of 3.55, 95\% CI of 1.43$8.84, P=.006$ (Table 5). Although multivessel disease was associated with ischemia in the univariable model (Table 4), this association was not preserved when 
Table 4. Plaque characteristics by CCTA associated with ischemia

\begin{tabular}{llclc}
\hline & OR & Standard error & $\boldsymbol{P}$ & $\mathbf{9 5 \%}$ CI \\
\hline Multiple vessels with stenosis $\geq 50 \%$ & $5.14^{\#}$ & 2.67 & .02 & $1.85-14.23$ \\
Non-calcified plaque & $4.89^{\#}$ & 3.83 & .043 & $1.05-22.73$ \\
Mixed plaque & 2.29 & 1.65 & .24 & $.55-9.41$ \\
Calcified plaque & 1.17 & .90 & .83 & $.25-5.31$
\end{tabular}

\#Univariate analysis describing increased odds of ischemia in patients with multiple vessels containing luminal stenosis $\geq 50 \%$, or non-calcified plaque. $P<.05$ is statistically significant. All patients with luminal stenosis $>0 \%$ were included in the analysis.

Table 5. Multivariable analysis of CCTA-based predictors of ischemia

\begin{tabular}{lcccc}
\hline & OR & Standard error & P & 95\% CI \\
\hline Age & 1.03 & .02 & .14 & $.99-1.07$ \\
Male & .79 & .38 & .62 & $.30-2.05$ \\
Diabetes & 1.34 & .45 & .38 & $.69-2.60$ \\
Hypertension & 2.10 & 1.04 & .13 & $.79-5.56$ \\
Smoking & .70 & .39 & .52 & $.23-2.09$ \\
Hyperlipidemia & .89 & .41 & .80 & $.35-2.23$ \\
Stenosis 50-89\% & $7.31 *$ & 4.22 & .001 & $2.35-22.70$ \\
Stenosis 90-100\% & $34.05^{*}$ & 20.26 & .0001 & $10.60-109.34$ \\
Serial stenoses $\geq 50 \%$ & $3.55^{*}$ & 1.65 & .006 & $1.43-8.84$ \\
Calcified plaque & .59 & .28 & .28 & $.22-1.53$ \\
Non-calcified plaque & 1.29 & .63 & .59 & $.49-3.38$ \\
Mixed plaque & 1.07 & .62 & .90 & $.34-3.36$ \\
Multiple vessels with stenosis $\geq 50 \%$ & 1.33 & .78 & .61 & $.42-4.23$ \\
\hline
\end{tabular}

${ }^{*}$ Multivariable analysis describing increased odds of ischemia in patients with stenosis severity $\geq 70 \%$, or with serial stenoses $\geq 50 \%$ by CCTA. $P<.05$ is statistically significant. All 292 patients were included in the analysis.

confounding variables such as stenosis severity, and plaque location were added to the model (Table 5).

\section{DISCUSSION}

In this head-to-head comparison of stenosis severity assessment by CCTA and myocardial perfusion by SPECT-MPI, we observe the following key findings. One, there is a direct relationship between luminal stenosis severity measured by CCTA and the prevalence of ischemia measured by SPECT-MPI. Two, at the extremes of luminal stenosis severity, $<50 \%$ (NPV of $98 \%$ ) or $\geq 90 \%$ (PPV of $74 \%$ ), CCTA capably predicts the absence or presence of ischemia. On the other hand, there is wide variability in the prevalence of ischemia among patients with a luminal stenosis severity of 50$90 \%$. Three, the presence of multiple plaques causing a stenosis $\geq 50 \%$ in the same vessel is strongly associated with ischemia on SPECT-MPI. To our knowledge, this is the first study showing that CCTA-based detection of serial lesions of $\geq 50 \%$ stenosis are more likely than single lesions to be associated with ischemia. Additionally, this is the first direct comparison of CCTA and automatic computer-based quantitation of SPECT-MPI in patients without a history of CAD to be reported from a single center.

Our results are similar to observations by other investigators who showed that $31-50 \%$ of all individuals with a maximal stenosis of more than $50 \%$ had ischemia with a prevalence of ischemia ranging from $16 \%$ to $20 \%$ in the subgroup of individuals with a maximal stenosis of $50-75 \% .^{7-12}$ When patients with coronary arteries containing dense calcium were excluded from analysis, we found an improvement in PPV and specificity of diagnosing ischemia. To more accurately reflect clinical decision making, we employed an intention-to-diagnose approach, and therefore included a secondary analysis in which segments with dense calcium were considered to have maximal stenosis. This approach has been used by other investigators, ${ }^{3,7-10}$ whose studies uniformly found that, when nonevaluable segments were scored as having maximal stenosis, sensitivity increased at the expense of specificity. 
Compared to a maximal stenosis threshold of $90 \%$, a $50 \%$ or a $70 \%$ maximal stenosis provide a significantly increased sensitivity and only a small decrease in specificity resulting in a greater area under the ROC curve. Based on the ROC analysis, the 50\% and 70\% stenosis cutoffs were similarly effective in discriminating between patients with and without ischemia on SPECT-MPI. While the 50-69\% category was not statistically different from the lesser degrees of stenosis with respect to the prevalence of ischemia, this category showed a trend toward increase in the prevalence and combined extent and severity of ischemia. The lack of statistical difference in this category may be due to the small sample size. Our findings are similar to what was reported by Di Carli et al in their comparison of stenosis severity by CCTA and myocardial ischemia by PET $^{12}$ and by Sato et al in their comparison of CCTA and SPECT. ${ }^{9}$ Careful examination of our SPECT-MPI results indicates that perfusion defects seen in 5 out of the 6 individuals with $<50 \%$ luminal stenosis by CCTA were most likely caused by factors unrelated to coronary artery stenosis. A 50\% stenosis threshold on CCTA may therefore provide a safe cutoff below which ischemia could be ruled out. Importantly, in a large sample of 114 arteries in 89 patients whose maximal stenosis was 25 $50 \%$, ischemia was not observed, implying that these patients do not need to be considered for ischemia testing. These findings are concordant with our recent observations that only $1.9 \%$ of $25-50 \%$ lesions by CCTA are associated with $>70 \%$ stenosis by invasive coronary angiography. ${ }^{20}$ Our observations highlight the importance of the complementary role of myocardial perfusion imaging in individuals with luminal stenosis of intermediate severity and the need for improving CCTA-based detection of myocardial ischemia beyond that available from assessment of stenosis severity. In patients with $\geq 90 \%$ stenosis and without dense calcification, the high prevalence of abnormal SPECT-MPI (74\%) suggests that it may be reasonable to assume such lesions are ischemia producing without needing further testing.

\section{Plaque Composition, Serial Stenoses, and Multivariable Predictors of Ischemia}

Previous studies have shown an association between non-calcified and partially calcified plaque with myocardial ischemia. ${ }^{32-35}$ In our analysis, there was a significant association of non-calcified plaque with ischemia in a univariable model; however, this association was not preserved when other confounding factors such as stenosis severity were considered in the multivariable model. Our analysis also revealed a strong relationship between multivessel $\geq 50 \%$ stenotic disease and ischemia that faded when accounting for the presence of high grade stenosis.

When we evaluated the relationship between serial stenoses and ischemia, we found that presence of multiple significant stenoses in the same vessel was strongly associated with ischemia on SPECT-MPI. The strong relationship between serial stenoses and ischemia remained even after adjustments for severity of individual luminal stenosis were made. This observation is consistent with the physiological effect of serial stenoses on resistance to coronary blood flow that has previously been described in both experimental animals and in patients with epicardial coronary artery disease. ${ }^{36,37}$ In the presence of serial stenoses, the net resistance to coronary blood flow is determined by the sum of the individual resistances and can result in greater stressinduced impairment of myocardial perfusion compared to a single stenosis with the same reduction in luminal diameter. ${ }^{36,37}$ The incremental value of detecting serial $\geq 50 \%$ stenosis when assessing coronary arteries by CCTA could potentially improve our ability to identify individuals likely to manifest inducible ischemia.

\section{LIMITATIONS}

Our studies were a retrospective analysis of patients who had undergone SPECT-MPI and CCTA within 6 months. Although this is a fairly long duration between studies, the median duration between studies was 4 days and $75 \%$ of patients underwent both studies within 2 weeks. Moreover, patients considered for analysis did not have a change in clinical status between the studies. Our study included a significant number of patients with low pretest likelihood of CAD and this may be reflected in the lower prevalence of ischemia (16\%) in our overall study population, compared to other studies in which the prevalence ranged between $40 \%$ and $50 \% .^{7-10}$ Our analysis does not use invasive angiography as the gold standard in comparison to CCTA-based stenosis assessment. However, stenosis severity defined anatomically has limitations in predicting functional significance since it does not account for collateral flow, vascular tone, or plaque geometry, variables that could potentially influence coronary blood flow. On the other hand, SPECTMPI offers an accurate assessment of the hemodynamic significance of coronary artery stenosis especially in lesions that may not appear to be severe by angiography and the prognostic significance of ischemia detected by SPECT-MPI has been well established. ${ }^{5,6}$ We have therefore chosen to compare CCTA-based stenosis to a functional assessment of coronary perfusion based on SPECT-MPI. SPECT-MPI may fail to detect ischemia in patients with three-vessel or left main disease due to a balanced reduction of flow and may therefore be 
suboptimal as a gold standard for ischemia. ${ }^{38}$ This may be better resolved in future studies where CCTA-based stenosis assessment is compared to absolute coronary blood flow and flow reserve measurements using PET myocardial perfusion.

While non-calcified plaque tended to be highly associated with ischemia compared to calcified plaque and serial stenoses tend to be more predictive of ischemia on SPECT compared to isolated stenoses of the same severity, the modest proportion of patients with ischemia $(16 \%)$ or with stenosis in the intermediate range of $50-70 \%(11 \%)$, limits the inclusion of other plaque characteristics such as positive remodeling, plaque volume, spotty calcification, or presence of large lipid core in a multivariable analysis. We suspect that consideration of additional CCTA-based imaging features of plaque assessment may be most useful in patients with intermediate-severity stenoses.

\section{CONCLUSIONS}

The presence of ischemia increases in proportion to the severity of luminal stenosis and the PPV of ischemia measured by SPECT-MPI rises as stenosis severity increases. A luminal stenosis of $\geq 90 \%$ by CCTA is a strong predictor of ischemia on SPECT-MPI. Individuals with luminal stenosis of $<50 \%$ are so unlikely to have myocardial ischemia that such patients may generally not need ischemia testing. In the group of individuals with luminal stenosis of $\geq 50 \%$, the presence of serial luminal stenoses of $\geq 50 \%$ may offer incremental value in identifying individuals at risk for ischemia.

\section{Open Access}

This article is distributed under the terms of the Creative Commons Attribution Noncommercial License which permits any noncommercial use, distribution, and reproduction in any medium, provided the original author(s) and source are credited.

\section{References}

1. Mowatt G, Cook JA, Hillis GS, Walker S, Fraser C, Jia X, et al. 64-Slice computed tomography angiography in the diagnosis and assessment of coronary artery disease: Systematic review and meta-analysis. Heart 2008;94:1386-93.

2. Budoff MJ, Dowe D, Jollis JG, Gitter M, Sutherland J, Halamert E, et al. Diagnostic performance of 64-multidetector row coronary computed tomographic angiography for evaluation of coronary artery stenosis in individuals without known coronary artery disease: Results from the prospective multicenter ACCURACY (Assessment by Coronary Computed Tomographic Angiography of Individuals Undergoing Invasive Coronary Angiography) trial. J Am Coll Cardiol 2008;52:1724-32.
3. Meijboom WB, Meijs MF, Schuijf JD, Cramer MJ, Mollet NR, van Mieghem CA, et al. Diagnostic accuracy of 64-slice computed tomography coronary angiography: A prospective, multicenter, multivendor study. J Am Coll Cardiol 2008;52:2135-44.

4. Miller JM, Rochitte CE, Dewey M, Arbab-Zadeh A, Niinuma H, Gottlieb I, et al. Diagnostic performance of coronary angiography by 64-row CT. N Engl J Med 2008;359:2324-36.

5. Hachamovitch R, Berman DS, Shaw LJ, Kiat H, Cohen I, Cabico $\mathrm{JA}$, et al. Incremental prognostic value of myocardial perfusion single photon emission computed tomography for the prediction of cardiac death: Differential stratification for risk of cardiac death and myocardial infarction. Circulation 1998;97:535-43.

6. Hachamovitch R, Hayes SW, Friedman JD, Cohen I, Berman DS. Comparison of the short-term survival benefit associated with revascularization compared with medical therapy in patients with no prior coronary artery disease undergoing stress myocardial perfusion single photon emission computed tomography. Circulation 2003;107:2900-7.

7. Schuijf JD, Wijns W, Jukema JW, Atsma DE, de Roos A, Lamb $\mathrm{HJ}$, et al. Relationship between noninvasive coronary angiography with multi-slice computed tomography and myocardial perfusion imaging. J Am Coll Cardiol 2006;48:2508-14.

8. Gaemperli O, Schepis T, Koepfli P, Valenta I, Soyka J, Leschka S, et al. Accuracy of 64-slice CT angiography for the detection of functionally relevant coronary stenoses as assessed with myocardial perfusion SPECT. Eur J Nucl Med Mol Imaging 2007; 34:1162-71.

9. Sato A, Hiroe M, Tamura M, Ohigashi H, Nozato T, Hikita H, et al. Quantitative measures of coronary stenosis severity by 64-Slice CT angiography and relation to physiologic significance of perfusion in nonobese patients: Comparison with stress myocardial perfusion imaging. J Nucl Med 2008;49:564-72.

10. Gaemperli O, Schepis T, Valenta I, Koepfli P, Husmann L, Scheffel $\mathrm{H}$, et al. Functionally relevant coronary artery disease: Comparison of 64-section CT angiography with myocardial perfusion SPECT. Radiology 2008;248:414-23.

11. Ravipati G, Aronow WS, Lai H, Shao J, DeLuca AJ, Weiss MB, et al. Comparison of sensitivity, specificity, positive predictive value, and negative predictive value of stress testing versus 64-multislice coronary computed tomography angiography in predicting obstructive coronary artery disease diagnosed by coronary angiography. Am J Cardiol 2008;101:774-5.

12. Di Carli MF, Dorbala S, Curillova Z, Kwong RJ, Goldhaber SZ, Rybicki FJ, et al. Relationship between CT coronary angiography and stress perfusion imaging in patients with suspected ischemic heart disease assessed by integrated PET-CT imaging. J Nucl Cardiol 2007;14:799-809.

13. Diamond GA, Forrester JS. Analysis of probability as an aid in the clinical diagnosis of coronary-artery disease. N Engl J Med 1979;300:1350-8.

14. Meijboom WB, van Mieghem CA, Mollet NR, Pugliese F, Weustink AC, van Pelt $\mathrm{N}$, et al. 64-slice computed tomography coronary angiography in patients with high, intermediate, or low pretest probability of significant coronary artery disease. J Am Coll Cardiol 2007;50:1469-75.

15. Flohr TG, McCollough CH, Bruder H, Petersilka M, Gruber K, Suss C, et al. First performance evaluation of a dual-source CT (DSCT) system. Eur Radiol 2006;16:256-68.

16. Dey D, Lee CJ, Ohba M, Gutstein A, Slomka PJ, Cheng V, et al. Image quality and artifacts in coronary $\mathrm{CT}$ angiography with dualsource CT: Initial clinical experience. J Cardiovasc Comput Tomogr 2008;2:105-14.

17. Gutstein A, Dey D, Cheng V, Wolak A, Gransar H, Suzuki Y, et al. Algorithm for radiation dose reduction with helical dual 
source coronary computed tomography angiography in clinical practice. J Cardiovasc Comput Tomogr 2008;2:311-22.

18. Ferencik M, Ropers D, Abbara S, Cury RC, Hoffmann U, Nieman $\mathrm{K}$, et al. Diagnostic accuracy of image postprocessing methods for the detection of coronary artery stenoses by using multidetector CT. Radiology 2007;243:696-702.

19. Raff GL, Abidov A, Achenbach S, Berman DS, Boxt LM, Budoff $\mathrm{M}$, et al. SCCT guidelines for the interpretation and reporting of coronary computed tomographic angiography. J Cardiovasc Comput Tomogr 2009;3:122-36.

20. Cheng V, Gutstein A, Wolak A, Suzuki Y, Dey D, Gransar H, et al. Moving beyond binary grading of coronary arterial stenoses on coronary computed tomographic angiography: Insights for the imager and referring clinician. JACC Cardiovasc Imaging 2008;1:460-71.

21. Achenbach S, Moselewski F, Ropers D, Ferencik M, Hoffmann U, MacNeill B, et al. Detection of calcified and noncalcified coronary atherosclerotic plaque by contrast-enhanced, submillimeter multidetector spiral computed tomography: A segment-based comparison with intravascular ultrasound. Circulation 2004;109:14-7.

22. Agatston AS, Janowitz WR, Hildner FJ, Zusmer NR, Viamonte M Jr, Detrano R. Quantification of coronary artery calcium using ultrafast computed tomography. J Am Coll Cardiol 1990;15:827-32.

23. Berman DS, Abidov A, Kang X, Hayes SW, Friedman JD, Sciammarella MG, et al. Prognostic validation of a 17-segment score derived from a 20 -segment score for myocardial perfusion SPECT interpretation. J Nucl Cardiol 2004;11:414-23.

24. Berman DS, Kang X, Hayes SW, Friedman JD, Cohen I, Abidov A, et al. Adenosine myocardial perfusion single-photon emission computed tomography in women compared with men. Impact of diabetes mellitus on incremental prognostic value and effect on patient management. J Am Coll Cardiol 2003;41:1125-33.

25. Nishina H, Slomka PJ, Abidov A, Yoda S, Akincioglu C, Kang X, et al. Combined supine and prone quantitative myocardial perfusion SPECT: Method development and clinical validation in patients with no known coronary artery disease. J Nucl Med 2006;47:51-8.

26. Germano G, Kavanagh PB, Su HT, Mazzanti M, Kiat H, Hachamovitch R, et al. Automatic reorientation of three-dimensional, transaxial myocardial perfusion SPECT images. J Nucl Med 1995;36:1107-14.

27. Slomka PJ, Nishina H, Berman DS, Akincioglu C, Abidov A, Friedman JD, et al. Automated quantification of myocardial perfusion SPECT using simplified normal limits. J Nucl Cardiol 2005;12:66-77.

28. Berman DS, Kang X, Gransar H, Gerlach J, Friedman JD, Hayes SW, et al. Quantitative assessment of myocardial perfusion abnormality on SPECT myocardial perfusion imaging is more reproducible than expert visual analysis. J Nucl Cardiol 2009;16:45-53.
29. Shaw LJ, Berman DS, Maron DJ, Mancini GB, Hayes SW, Hartigan PM, et al. Optimal medical therapy with or without percutaneous coronary intervention to reduce ischemic burden: Results from the Clinical Outcomes Utilizing Revascularization and Aggressive Drug Evaluation (COURAGE) trial nuclear substudy. Circulation 2008;117:1283-91.

30. Slomka PJ, Cheng VY, Dey D, Woo J, Ramesh A, Van Kriekinge $\mathrm{S}$, et al. Quantitative analysis of myocardial perfusion SPECT anatomically guided by coregistered 64-slice coronary CT angiography. J Nucl Med 2009;50:1621-30.

31. Hanley JA, McNeil BJ. A method of comparing the areas under receiver operating characteristic curves derived from the same cases. Radiology 1983;148:839-43.

32. Hoffmann U, Moselewski F, Nieman K, Jang IK, Ferencik M, Rahman AM, et al. Noninvasive assessment of plaque morphology and composition in culprit and stable lesions in acute coronary syndrome and stable lesions in stable angina by multidetector computed tomography. J Am Coll Cardiol 2006;47:1655-62.

33. Leber AW, Knez A, von Ziegler F, Becker A, Nikolaou K, Paul S, et al. Quantification of obstructive and nonobstructive coronary lesions by 64-slice computed tomography: A comparative study with quantitative coronary angiography and intravascular ultrasound. J Am Coll Cardiol 2005;46:147-54.

34. Leber AW, Knez A, White CW, Becker A, von Ziegler F, Muehling $\mathrm{O}$, et al. Composition of coronary atherosclerotic plaques in patients with acute myocardial infarction and stable angina pectoris determined by contrast-enhanced multislice computed tomography. Am J Cardiol 2003;91:714-8.

35. Lin FY, Saba S, Weinsaft JW, Wong FJ, Szulc M, Kligfeld P, et al. Relation of plaque characteristics defined by coronary computed tomographic angiography to ST-segment depression and impaired functional capacity during exercise treadmill testing in patients suspected of having coronary heart disease. Am J Cardiol 2009;103:50-8

36. Seiler C, Kirkeeide RL, Gould KL. Basic structure-function of the epicardial coronary vascular tree-the basis of quantitative coronary arteriography for diffuse coronary artery disease. Circulation 1992;85:1987-2003.

37. Kirkeeide RL, Gould KL, Parsel L. Assessment of coronary stenoses by myocardial perfusion imaging during pharmacologic coronary vasodilation. VII. Validation of coronary flow reserve as a single integrated functional measure of stenosis severity reflecting all its geometric dimensions. J Am Coll Cardiol 1986;7:103-13.

38. Berman DS, Kang X, Slomka PJ, Gerlach J, Yang LD, Hayes SW, et al. Underestimation of ischemia by gated SPECT myocardial perfusion imaging in patients with left main coronary artery disease. J Nucl Cardiol 2007;14:521-28. 